\title{
Frontières
}

\section{Tenir compte de la ritualité}

\section{Un changement organisationnel important pour une entreprise} funéraire

\section{Nicole Bouchard}

Volume 18, numéro 1, automne 2005

Hélas, célébrer la mort!

URI : https://id.erudit.org/iderudit/1074315ar

DOI : https://doi.org/10.7202/1074315ar

Aller au sommaire du numéro

\section{Éditeur(s)}

Université du Québec à Montréal

ISSN

1180-3479 (imprimé)

1916-0976 (numérique)

Découvrir la revue

Citer cet article

Bouchard, N. (2005). Tenir compte de la ritualité : un changement organisationnel important pour une entreprise funéraire. Frontières, 18(1),

51-56. https://doi.org/10.7202/1074315ar
Résumé de l'article

Cet article traite des différentes avenues empruntées par une entreprise funéraire pour être en phase avec les besoins des familles endeuillées et montre la nécessité du renouvellement des pratiques funéraires.
Ce document est protégé par la loi sur le droit d'auteur. L'utilisation des services d'Érudit (y compris la reproduction) est assujettie à sa politique d'utilisation que vous pouvez consulter en ligne.

https://apropos.erudit.org/fr/usagers/politique-dutilisation/ 


\section{Résumé}

Cet article traite des différentes avenues empruntées par une entreprise funéraire pour être en phase avec les besoins des familles endeuillées et montre la nécessité du renouvellement des pratiques funéraires.

Mots clés: ritualité - funérailles entreprise funéraire.

\section{Abstract}

This article presents the evolution of funeral practices in a single funeral home. The different propositions given by the author show the necessity of a renewal in the funeral rituals.

Keywords: rituality - funeral funeral home.

\section{TENIR COMPTE DE LA RITUALITÉ UN CHANGEMENT ORGANISATIONNEL IMPORTANT POUR UNE ENTREPRISE FUNÉRAIRE}

Nicole Bouchard, Ph.D. ,Théologie pratique, professeure au Département des sciences humaines et directrice du Laboratoire d'expertise et de recherche en anthropologie rituelle et symbolique (LERARS) à I'UQAC.

Dans la réflexion qui a cours sur la recomposition des rituels liés au passage de la mort, les entreprises funéraires sont en voie de devenir les acteurs pivots de plusieurs étapes du processus rituel. Il n'y a pas si longtemps leur rôle se concentrait principalement sur l'administration des aspects plus «matériels» de la mort. Aujourd'hui, la gestion du sens est désormais intégrée à l'offre de services et les professionnels œuvrant dans ce milieu sont en phase de devenir les «metteurs en sens» (St-Onge, 2001) privilégiés de ce passage. Un exemple qui métaphorise ce changement est sans nul doute la construction de complexes funéraires intégrant désormais une "chapelle» et une offre différenciée et personnalisée de services en matière de ritualité. Désormais on inscrit dans un espace donné un nouveau «corps» de professionnels et de nouveaux «corpus» rituels.

Il va sans dire que cette problématique a été rendue possible par l'élargissement de notre conception du champ religieux et l'autonomisation du spirituel à l'intérieur même de ce champ ${ }^{1}$. De plus en plus de familles souhaitent des rituels qui fournissent un espace pour l'expression de leur parcours de vie, leurs valeurs, leurs appartenances et leurs croyances. Comment ces demandes sont-elles gérées dans le quotidien des entreprises? Qui prend en charge l'accompagnement et la présidence de ces rituels? Cette pratique est-elle en train d'accélérer ou de colmater la tendance lourde de notre culture à diminuer le temps consacré au deuil? Quel partenariat les entreprises mettent-elles en place avec les églises?

Peu d'études praxéologiques ont été faites sur ce changement qui est en voie de modifier les représentations sociales de la mort. Les études plus théoriques s'inquiètent des impacts de ces nouveaux rituels «à la carte». Leurs questionnements se concentrent principalement sur les impacts de ces rituels sur la dé-socialisation de la mort (Thomas, 1985; Bacqué, 1997; Des Aulniers, 1991). L'ampleur et la portée des diagnostics, maintes fois relevés dans la littérature scientifique, dévoilent l'urgence de développer des partenariats de recherche et de formation pour accompagner et 
guider les entreprises dans ce changement qui va plus loin que l'embauche d'un personnel spécialisé, l'investissement dans la conception de kits rituels sur mesure ou la construction de chapelles.

Nous nous proposons ici de faire un premier bilan d'une pratique de rechercheaction-formation en cours dans la région du Saguenay-Lac-Saint-Jean depuis janvier 2005. Cette expérience regroupe une équipe de chercheurs de l'UQAC, spécialisée dans le champ de la ritualité, ainsi que les dirigeants et les employés de la Coopérative funéraire de Chicoutimi. La visée de cette intervention, de type exploratoire, consiste à instaurer une pratique réflexive devant conduire à une prise en charge responsable de la ritualité dans cette entreprise. Notre approche résolument praxéologique s'est organisée autour du comment, du qui et du où de cette pratique. Comment accompagner ce passage pour qu'il ne soit pas l'objet ou le résultat des seules lois régissant le marché? Comment soutenir les entreprises pour assumer cette tâche avec compétence? Comment instaurer une séquence rituelle signifiante et aidante pour les personnes touchées par la mort d'un proche? Qui de l'église ou de l'entreprise assumera les différentes tâches liées à la présidence de rituels? Doit-on construire une «chapelle» pour soutenir une telle pratique? Tels ont été les premiers questionnements qui orientent le présent compte rendu. Au stade actuel de notre démarche, il s'agit de procéder à une première relecture de l'itinéraire parcouru. La poursuite de nos travaux devrait nous permettre de systématiser les étapes d'un processus de changement qui intégrerait au sein même des entreprises le nécessaire travail d'éducation à la mort et l'accompagnement du sens.

\section{AU DÉPART DE LA PRATIQUE: UN SENTIMENT D'URGENCE DE LA PART DES DIRIGEANTS DE L'ENTREPRISE}

L'aventure a débuté par un coup de fil au bureau. La demande est précise et directe. Elle émane des dirigeants de l'entreprise, à savoir le directeur et son adjointe, et elle se formule ainsi: "Nous connaissons bien les travaux que vous effectuez dans le champ de la ritualité et nous aimerions vous rencontrer pour travailler avec vous. » Un rendez-vous fut fixé dans la même semaine. La première rencontre dura environ une heure trente minutes et j'en garde un souvenir très prégnant. J'ai noté dans mon journal de bord mon étonnement devant la connaissance que ces praticiens avaient des enjeux entourant la ritualité funéraire. Ils se sont montrés préoccupés par les modifications rapides des habitudes de vie des familles en ce qui a trait à la gestion de la mort. Ils se questionnent sur les impacts de la disparition progressive des temps de symbolisation de la mort et sur le vécu du deuil. Ils se demandent si, à l'instar de leurs collègues des autres entreprises, ils doivent aller de l'avant dans le projet de construction d'une chapelle. Ils s'interrogent sur les possibilités de collaboration avec les autorités ecclésiales qui, pour des raisons souvent de manque de personnel, semblent de plus en plus se désintéresser de la pratique. Je me permets de citer quelques phrases qui ont marqué cette première rencontre.

Nous voulons que vous nous aidiez à intervenir auprès des familles. Nous avons de plus en plus de demandes diversifiées et nous ne sommes pas suffisamment outillés pour animer des moments d'échange et d'intériorité. Comment assumer ce rôle avec encore plus de compétence.

Dirigeant $A$

Nous sommes conscients des effets néfastes sur le vécu du deuil des formules plus populaires qui réduisent au minimum le temps d'exposition. Comment mieux guider les familles et les aider à discerner ce qui est le meilleur pour eux?

\section{Dirigeant A}

Les familles ont tendance à aller très vite et à faire des choix qui diminuent toujours la prise de contact avec le corps du défunt. Nous savons que cela a des impacts sur le vécu du deuil mais comment questionner ces choix et aller au-delà des idées toutes faites et des préjugés.

\section{Dirigeant $B$}

Nous avons un répertoire de prières et de textes de réflexion, le personnel est très proche et prend beaucoup de temps avec les familles mais plusieurs de nos employés aimeraient être encore mieux outillés. Nous souhaiterions élargir notre coffre à outils pour bonifier nos interventions.

\section{Dirigeant B}

Nous avons des demandes de plus en plus nombreuses pour des célébrations ici au salon. Nous n'avons pas de lieu comme une chapelle pour mieux vivre ces demandes. Pourquoi construire un tel lieu? Y a-t-il des éléments dont nous devons tenir compte pour bien orienter ce projet important?

\section{Dirigeant $B$}

C'est à partir de tels énoncés que nous avons travaillé avec les dirigeants de l'entreprise pour établir précisément la teneur de leur demande. Notre visée est de permettre une meilleure intégration de la dimension rituelle dans l'ensemble des activités de l'entreprise. Nous sommes convaincus que c'est à toutes les étapes du processus d'intervention que la préoccupation herméneutique doit être présente. Ainsi, il ne s'agit pas de saupoudrer ici et là des moments rituels déjà construits ou encore de fournir un répertoire de célébrations pouvant être reproduites et recopiées par le personnel. Pour l'entreprise, c'est de changement qu'il était question, un changement visant à introduire dans le quotidien de la tâche les enjeux liés à la gestion du sens par et à travers le processus rituel. Cet aspect de la demande nous a séduits dans la mesure où elle évite de penser la ritualité comme séparée et indépendante des autres réalités de l'entreprise. Notre implication depuis quelques années à l'égard de cette pratique nous a permis de constater que le travail du sens ne consiste pas à faire plus, mais bel et bien à faire autrement. Il s'agit d'une «conversion» du regard qui modifie l'ensemble des actions menées par le personnel. Et c'est souvent là que le bât blesse car plusieurs entreprises hésitent à prendre un tel virage qui modifie l'organisation même du travail. C'est donc dans l'esprit de ce consensus que nous avons débuté à l'hiver 2005 notre démarche. Quel chemin avons-nous suivi? Quelles ont été les premières étapes de notre parcours? Quels outils ou moyens de formation avons-nous mis de l'avant pour atteindre nos objectifs? Autant de questions que nous aborderons maintenant.

\section{LES PREMIÈRES ÉTAPES DU PROCESSUS DE CHANGEMENT}

PHASE D'APPRIVOISEMENT:

LE TRAVAIL AVEC L'ENSEMBLE

DU PERSONNEL DE L'ENTREPRISE

Il nous a semblé important que le consensus partagé par les dirigeants de l'entreprise et l'équipe de recherche devienne une problématique portée par l'ensemble $\mathrm{du}$ personnel. Ainsi la première phase de nos travaux a consisté à créer un espace de dialogue. Des séances d'échange, quatre journées au total, ont eu lieu. Il n'y a pas d'agenda caché, un seul sujet à l'ordre du jour: comment vivent-ils au quotidien avec les demandes de plus en plus différenciées des familles en matière de ritualité (prières, réflexions, échanges, etc.). Que font-ils pour répondre à ces demandes? Quelles sont les difficultés qu'ils éprouvent? Les rencontres se vivent dans une atmosphère de détente et de questionnement. Les membres du personnel amènent leurs interrogations sur le sens de ce changement et veulent savoir comment leur tâche s'en trouvera modifiée. Il convient de regrouper quelques-uns des points saillants de nos échanges. 
REDÉCOUVRIR L'IMPORTANCE

DE LA DIMENSION ANTHROPOLOGIQUE

\section{DES RITUELS}

Certains membres du personnel éprouvent un malaise à s'intéresser à la ritualité et à s'y impliquer. Pour ces derniers, le travail du «sacré» doit reposer sur des personnes « consacrées». Ainsi quelques-uns hésitent à s'impliquer dans la pratique de rituels parce que, selon eux, cette fonction est du ressort du clergé. Ils considèrent les religions institutionnelles comme les seuls porteurs autorisés du travail de sens. Dès lors, les efforts de notre équipe devraient se concentrer sur une re-négociation avec le personnel des églises qui possèdent la formation autorisée pour présider un rituel. Il nous a ainsi fallu travailler avec le personnel et leur fournir des grilles de compréhension de la pratique précisant l'autonomie anthropologique des rituels et la possibilité de les détacher des rituels religieux.

\section{ÉVITER DE CONCENTRER}

LA DÉMARCHE RITUELLE

SUR UN OU DEUX MOMENTS FORTS DE LA PRATIQUE

Les rituels se résument souvent à quelques moments forts de l'intervention et quelquefois à la seule célébration d'adieu. On associe le travail du sens et de symbolisation à la seule célébration des funérailles ou d'un adieu. De l'ancienne route des morts, nous n'aurions conservé que ce dernier moment de séparation. Quant aux autres rituels liés à la période de marge ou d'agrégation ${ }^{2}$, ils se perdent et s'évanouissent dans une série de gestes et de paroles souvent sans direction. Le travail de sens est disséminé et l'histoire de vie des familles et leurs représentations de la mort et de l'après-vie trouvent peu d'échos et font l'objet de peu de réinvestissements. Comment permettre le déploiement, la maturation du récit de vie des familles afin qu'il puisse être mis en scène à travers des gestes, des symboles, des mots susceptibles de former un réservoir suffisamment riche pour alimenter la longue et difficile traversée du deuil? Nous avons là un dossier important qui ouvre un chantier majeur de formation.

\section{FAVORISER ET SOUTENIR}

\section{DES LIEUX SÉCULIERS DE CÉLÉBRATION}

Limportance d'avoir un lieu pour célébrer les différents rituels est ressortie. Les rites doivent permettre la coupure, l'établissement de frontières et de limites. L'aménagement d'un lieu capable de favoriser l'intériorité et susceptible d'offrir un environnement symboliquement riche est apparu comme une urgence. C'est ainsi que nous avons travaillé en étroite collaboration avec les responsables de l'entreprise afin

POUR L'ENTREPRISE, C'EST DE CHANGEMENT QU'IL ÉTAIT QUESTION, UN CHANGEMENT VISANT À INTRODUIRE DANS LE QUOTIDIEN DE LA TÂCHE LES ENJEUX LIÉS À LA GESTION DU SENS PAR ET À TRAVERS LE PROCESSUS RITUEL.

d'alimenter le travail du maître verrier et de l'architecte retenus pour le projet. Ce lieu se voulait un «atelier» où l'on met à la disposition des membres du personnel un environnement susceptible de soutenir le travail d'intériorité et de créativité nécessaire à la performativité rituelle.

L'analyse de ces premières observations nous a permis de retenir deux objectifs susceptibles d'orienter nos interventions. Le premier tourne autour de la formation à dispenser au personnel pour mieux développer leur compétence en matière de ritualité et l'autre, plus immédiat, concerne les enjeux entourant la construction d'un lieu de célébration.

\section{VERS L'ÉLABORATION D'UN PROJET DE FORMATION CONTINUE, INTÉGRÉE AU VÉCU DE L'ENTREPRISE}

Les besoins du personnel en formation sont diversifiés et importants. Par où commencer? Comment déjà enclencher le changement à court terme mais aussi permettre le développement de compétences en matière d'accompagnement et de gestion du sens. Il fallait d'abord prendre quelques mesures transitoires pour gérer les demandes des familles et accompagner au quotidien le personnel. C'est ainsi que nous avons élaboré un Cahier des charges et mis en place une formule d'accompagnement individuel en entreprise (coaching). Parallèlement à cet investissement à court terme, nous devions nous assurer que les membres du personnel chargés du contact avec les familles et intéressés à développer leurs compétences d'accompagnement puissent amorcer un processus de formation plus systématique. C'est dans cette optique que nous les avons invités à se joindre, en septembre 2005 , à la première cohorte d'étudiants inscrits à notre Programme court de premier cycle en intervention rituelle.

\section{ASSURER UN CONTINUUM DE SENS}

Nos observations et échanges avec les dirigeants et le personnel de l'entreprise ont permis de constater que la Coopérative funéraire de Chicoutimi possédait déjà une organisation de travail qui permettait un bon suivi des familles. Ainsi, l'hôtesse qui accueille la famille pendant la période d'exposition est souvent présente lors de la célébration d'adieu et des autres moments rituels (incinération, mise en niche ou en terre, célébration commémorative, etc.). Il y avait déjà là des conditions qui, au plan de la gestion du temps, facilitaient, notre intervention. Par ailleurs, afin d'introduire dans la pratique des intervenants le souci permanent de la construction de sens, il fallait fournir des outils pour permettre de recueillir le récit de la famille et par la suite de le transmettre aux différentes personnes susceptibles d'entrer en contact avec cette famille (conseiller et conseillère, hôtesse, directeur des funérailles, président de la célébration d'adieu, etc.).

Il a été convenu qu'un dossier serait ouvert pour chacune des familles. Ce dossier contient des informations sur le sens que les proches donnent à l'événement, la nature des liens qui unissent entre eux les membres de la famille, les grands thèmes qui traversent et caractérisent la vie de la personne décédée. C'est ainsi que, dès l'entrevue d'accueil, s'ajoutent à la démarche habituelle des questions qui permettent de recueillir le matériel discursif et symbolique nécessaire à l'élaboration des rituels. Voici un court extrait des indications formulées à cet égard à l'intention de la personne responsable de l'entrevue d'accueil.

Nous suggérons aux conseillers et conseillères d'ajouter à leur protocole d'entrevue les questions suivantes:

- Des images, des symboles qui représentent la personne décédée.

- Une chanson, un poème, une œuvre (si la personne avait des habiletés artistiques), une phrase représentative de la vie de la personne (ou qu'elle affectionnait).

- Des gestes que vous trouvez important de poser? Des personnes dans votre famille qui pourraient prendre la parole?...

Ces informations seront consignées sur une fiche synthèse qui sera remise à l'hôtesse responsable de la famille pour qu'elle puisse, dès les premiers temps de l'exposition, avoir une intervention adaptée et en continuum avec ce premier contact.

Nous avons fait un travail d'élaboration pour chacun des moments et des acteurs impliqués auprès des familles. L'outil n'est pas finalisé mais nous comptons terminer 
l'élaboration de ce dossier de travail au cours de la prochaine année. Dans l'immédiat, nous ne disposons pas encore de données sur les impacts de cet outil mais la poursuite de nos travaux devrait nous permettre d'évaluer les résultats d'une telle mise en œuvre sur le continuum rituel.

\section{METTRE EN PLACE}

\section{UNE FORMULE DE COACHING}

À côté de ce volet lié à l'organisation concrète du travail, nous avons mis en place une modalité de formation souple, capable de répondre aux demandes des personnes qui, au sein de l'entreprise, ont démontré leur intérêt à bonifier leur compétence dans le champ de l'intervention rituelle. Cette modalité de formation souple, que nous appelons coaching, nous permet d'intervenir sur des problématiques dans l'ici et maintenant de la pratique et en tenant compte des demandes des familles. Il ne s'agit pas de faire des familles et du personnel des cobayes mais de favoriser l'apprentissage par l'expérience pour le développement du savoir-faire. Nous cherchons à guider les personnes dans l'exercice de leurs fonctions. Ce coaching sera assuré par une étudiante finissante à la maîtrise en théologie pratique qui possède une solide expertise dans le champ de l'intervention rituelle. Concrètement, l'accompagnateur (coach) ne se substitue pas au personnel. Il ne fait pas le travail à leur place; il a un rôle de soutien, d'écoute, d'accompagnement. Le coach est là pour soutenir la démarche rituelle et pour veiller à sa progression. Quelquefois, il aidera à trouver les outils qui vont permettre au personnel de risquer une parole et d'expérimenter les enjeux liés à la présidence rituelle. Cette expérience a débuté en septembre 2005 à raison d'une journée par semaine environ.

\section{OFFRIR UNE FORMATION UNIVERSITAIRE \\ DANS LE CHAMP \\ DE L'INTERVENTION RITUELLE}

Dès le départ, nous avons insisté sur le fait que les formules de formation à court terme sont insuffisantes pour permettre à des personnes d'intégrer avec aisance les compétences nécessaires à la mise en scène rituelle. Nous avons donc travaillé à l'élaboration d'un programme de formation de niveau universitaire qui permet de développer les habiletés, attitudes et savoirs liés à l'intervention rituelle. Le programme a débuté en septembre 2005 et quatre membres du personnel de l'entreprise sont inscrits à cette formation. Le cours est offert principalement aux personnes impliquées dans l'une ou l'autre étape du passage de la mort. En tout, une vingtaine de personnes y sont déjà inscrites provenant de milieux de la santé, de milieux communautaires et des

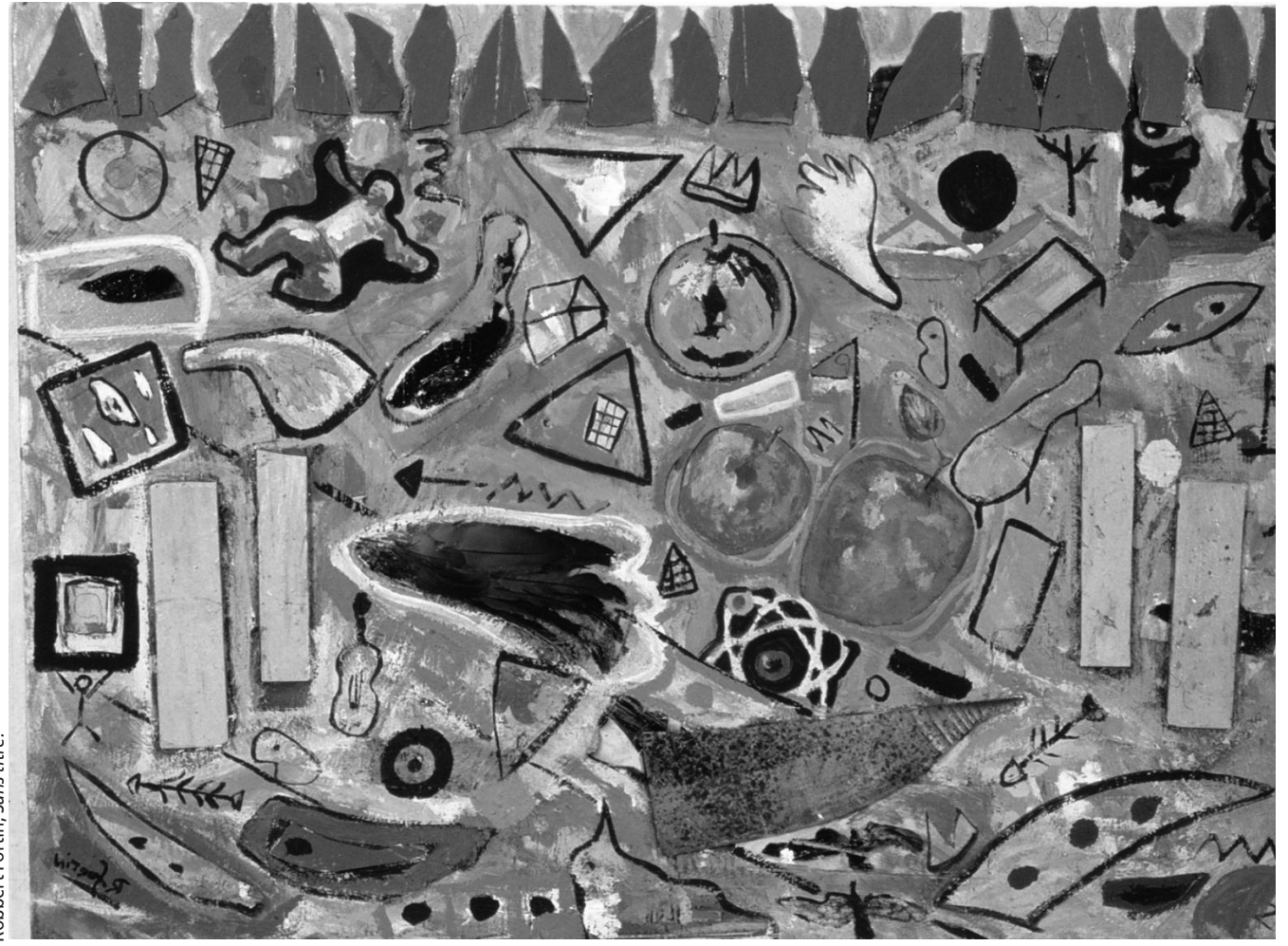

entreprises funéraires. Nous avons ainsi une cohorte qui couvre l'ensemble des étapes de la nouvelle route des morts au sein de notre culture (maisons de soins palliatifs, entreprises funéraires, communautés chrétiennes, groupes communautaires, consultants psychosociaux en cabinets privés). Les objectifs et la visée de ce programme court de 12 crédits sont les suivants:

Le programme court en intervention rituelle et symbolique de l'UQAC souhaite répondre aux besoins de formation des personnes qui sont en contact quotidien avec des hommes et des femmes vivant un passage difficile. S'adressant aux personnes désireuses de s'impliquer dans une démarche qui leur permet d'intervenir en utilisant la richesse de la structure des rites de passage pour accompagner la quête de sens des femmes et des hommes d'ici, ce programme novateur et original vise à développer plusieurs compétences :

- Établir les forces et faiblesses des structures rituelles déployées dans le cadre de certains événements (décès, maladie, naissance, engagement, etc.);

- Intégrer les cadres de l'intervention rituelle et symbolique dans une pratique tant personnelle que professionnelle;

- Concevoir une intervention à partir de la ritualité et du symbolisme relatifs à certains événements;

- Approfondir la structure des rites de passage;
- Améliorer ses connaissances à l'égard des grands contenus de la sagesse humaine et spirituelle.

Ce programme de premier cycle s'article autour de quatre cours: Sociétés, rites et symboles; Le symbolisme et les passages de la vie; La mort et ses représentations; Projet d'intervention. Nous sommes conscients qu'il s'agit là du minimum requis pour intervenir dans le vaste champ de la ritualité humaine. Il faut souhaiter que nous puissions étendre à d'autres acteurs et à d'autres entreprises québécoises ce bref parcours de formation, qui se veut le premier moment d'une démarche que nous aimerions voir se déployer et se structurer d'une manière plus importante. On pense ici à un programme de formation initiale sous la forme d'un certificat, voire d'un baccalauréat pour des intervenants intéressés à se doter d'une compétence reconnue dans le champ de la ritualité et des passages de la vie.

\section{UNE PRÉOCCUPATION IMPORTANTE: UN LIEU ET UN TEMPS POUR CÉLÉBRER}

Une question a émergé en cours de route concernant la pertinence ou non d'avoir un lieu spécifique de célébration. En effet, l'expérience que nous avons eue avec différents milieux de pratique montre souvent que l'arrivée d'une chapelle modifie en fait très peu la manière dont les rituels sont élaborés et construits. L'objectif se limitant souvent à offrir une formule "tout en un» ou un guichet unique pour les familles. Comment aller au-delà de l'aménagement 
des horaires et de la facilitation des déplacements? Comment profiter de la création de cet espace nouveau pour différencier les démarches et offrir aux familles des choix en matière de ritualité qui dépassent le seul accommodement? Telles ont été les questions qui ont marqué le point de départ de notre réflexion plus liée aux lieux de la célébration, mais qui, en fait, concerne l'intégration de la démarche rituelle et son inscription dans un lieu précis au sein de l'entreprise. Comment avons-nous abordé ce projet, quelles en ont été les prémisses? Comment gérer et différencier les demandes en matière de ritualité? C'est ce que nous nous attacherons à décrire.

\section{LA CHAPELLE DE LA $5^{\mathrm{e}}$ SAISON: UN LIEU PLUS SÉCULIER DE CÉLÉBRATION}

Afin de sortir du cadre trop étroit qu'aurait été une réflexion sur la seule utilité d'un lieu autonome de célébration, un premier exercice a été réalisé autour du nom à donner à ce lieu. Cette démarche a permis un travail de création et tous se sont mis d'accord pour appeler ce lieu: la Chapelle de la $5^{\mathrm{e}}$ Saison. Le mot chapelle a fait problème, mais faute de mieux nous l'avons conservé. Un texte a été rédigé pour mettre en évidence le sens de cette nomination. Ce texte est reproduit dans le feuillet qui présente les nouveaux services désormais offerts par la Coopérative funéraire de Chicoutimi.

\section{LA CHAPELLE DE LA 5e SAISON}

C'est un espace de silence et de recueillement

où on prend le temps de dire un dernier adieu

à ceux et celles qui nous quittent toujours trop tôt,

dans la force de leur été ou à l'automne de leur vie.

C'est un temps d'arrêt où on cherche ensemble

les mots, les gestes, les chants pour traverser le froid de l'hiver qui nous transperce quand survient la mort.

C'est un moment où on se laisse toucher par des rites,

des symboles qui disent la force tenace de notre espérance et de notre quête.

C'est un lieu de passage, une fenêtre sur l'autre rive,

qui permet à ceux et celles qui vivent un deuil

de partager leurs peurs et leurs angoisses

dans la confiance d'une promesse d'un printemps toujours renouvelé.
La Chapelle de la $5^{\text {e }}$ Saison, c'est beaucoup plus qu'un lieu.

C'est un jardin où l'on cherche

à faire croître la vie

malgré la dureté des recommencements.

Elle s'enracine dans la promesse

de nos hivers,

elle bourgeonne à la sève

de nos printemps,

elle fleurit à l'arbre de nos étés,

elle livre ses fruits dans la paix

de nos automnes.

Au-delà du lieu c'est un climat de paix, une terre d'accueil,

pour ceux et celles qui souffrent de l'absence.

Nous avons aussi collaboré à la conception du lieu en proposant la symbolique qui va alimenter la construction des rituels. Architecte et artiste verrier ont ainsi créé un environnement qui inscrit la traversée dans une perspective nettement anthropologique. Un arbre est planté à l'avant et constitue pour l'instant l'élément essentiel de la scène rituelle. Cet arbre de vie est aussi entouré d'une série de quatre vitraux qui sont créés à partir des différents éléments qui soutiennent et rendent possible la vie: l'eau, la terre, le feu. Un extrait du commentaire de l'artiste verrier illustre bien le travail qui a été accompli:

C'est à partir de la symbolique et des thèmes qu'on m'a proposés, qui sont l'eau, le feu et l'arbre que j'ai réalisé mes dessins qui vont ensuite servir aux plans pour réaliser l'œuvre finale. Le thème de la vie, omniprésent dans la rencontre de ces trois éléments, est l'essence même de ce concept. Ainsi, la représentation de la nature n'est pas là simplement pour produire un effet bucolique, mais bien pour établir une identité avec cette $5^{\text {e }}$ Saison qu'on suppose dans une autre dimension spirituelle. La difficulté, c'est de réussir à placer l'observateur dans un état d'esprit qui invite à la réflexion et prédispose à une vision intériorisée de son imaginaire. Les éléments architecturaux des planchers, des colonnes et des stèles de feu qui sont intégrés, accentuent cet effet de dimension et de profondeur et encadrent ce qui semble être cette porte qui s'ouvre sur la lumière de cette autre vie ${ }^{3}$.

\section{UN ESPACE DE DIFFÉRENCIATION DES CROYANCES}

Dès le début de notre collaboration, il a été convenu qu'il nous fallait innover au plan de la pratique tout en conservant un partenariat de qualité avec les églises paroissiales. En effet, dans la région du Saguenay-Lac-Saint-Jean, les églises et les entreprises funéraires sont des partenaires de longue date. Comment introduire ce changement sans que le partenaire ecclésial se sente hors, voire «ob » scène? C'est ainsi que l'entreprise a profité de l'événement marquant la construction de la chapelle pour jeter les bases d'un partenariat plus différencié avec les églises paroissiales.

Nous avons bien précisé que ce lieu ne veut pas se substituer aux églises paroissiales mais qu'il s'offre plutôt comme un espace où d'autres avenues seront explorées. Notre pratique va bien au-delà d'une offre de célébration d'adieu ou d'une pâle reprise des funérailles chrétiennes. Sans exclure le moment des funérailles, nous ne nous concentrons pas sur ce seul événement; la possibilité qu'offre ce lieu de redéployer un continuum rituel signifiant. Il s'agit d'un espace qui, nous l'espérons, va permettre de réintroduire une séquence rituelle, de déployer la fonction d'éducation à la mort, fonction qui échoit désormais aux professionnels œuvrant dans les entreprises funéraires. Un extrait du dépliant d'information décrivant les services qui seront offerts à l'intérieur de la chapelle confirme la volonté de diversifier les possibilités d'utilisation de ce lieu.

\section{LA CÉLÉBRATION D'ADIEU}

C'est une célébration de la parole qui se vit à la résidence funéraire, en partenariat avec la communauté paroissiale. Les symboles et les rites qui sont vécus dans cette cérémonie sont inspirés des célébrations religieuses traditionnelles mais sans la présence de l'eucharistie. La famille peut utiliser les services d'un prêtre, d'un diacre ou d'un agent de pastoral laïc pour présider la célébration.

\section{LE RITUEL DE MÉMOIRE}

Cette célébration célèbre la vie de la personne décédée à l'aide de textes, de chansons, de rites ou de symboles très signifiants pour la famille. C'est la maison funéraire qui assure la présidence de la cérémonie. Les personnes désignées pour coordonner le Rite d'adieu sont formées pour répondre aux besoins des familles endeuillées.

\section{LE RITUEL D'ANNIVERSAIRE}

On pourra souligner et célébrer les anniversaires d'un décès lors d'un rituel approprié aux demandes de la famille.

\section{LE RITUEL POUR LES ENFANTS}

Il existe maintenant des rituels pour accompagner, préparer et aider les enfants à entrer en contact avec la dépouille d'un proche.

Il s'agit, bien sûr, d'un premier travail de diversification de la pratique. D'autres avenues seront explorées dans le cadre de 
notre partenariat de recherche. Ce qu'il faut retenir, c'est la prise en charge de la ritualité par l'entreprise. Un tel modèle constitue une alternative à la pratique actuelle qui souvent mise sur l'embauche d'un personnel spécialisé et ad hoc moins bien intégré à la vie de l'entreprise. Notre intervention vient trancher avec un modèle dont la tendance lourde confine le champ de la ritualité à celui du religieux, comme si ce dernier en avait l'exclusivité.

C'est avec beaucoup d'humilité que nous avons présenté les premiers moments de notre partenariat avec la Coopérative funéraire de Chicoutimi. Cette recherche praxéologique en est à ses premiers pas mais nous sommes particulièrement fiers d'avoir créé un espace de réflexion et de dialogue avec ce milieu de pratique. Bien du travail reste à accomplir mais nous pensons avoir réuni les conditions pour lancer un projet durable, la confiance et le respect mutuel étant les ingrédients nécessaires à toute avancée de la réflexion. Comme chercheure, ce projet m'a permis de côtoyer et de bien comprendre le travail des personnes œuvrant dans ce champ de pratique. Nous avons pu dépasser les préjugés qui trop souvent empêchent la création d'un espace de dialogue et paralysent bien des efforts de renouveau. Nous avons rencontré des personnes soucieuses d'accompagner les familles avec la ferme volonté de bonifier
LE TRAVAIL DU SENS

NE CONSISTE PAS À FAIRE PLUS,

MAIS BEL ET BIEN

À FAIRE AUTREMENT.

leur approche dans la ligne d'un développement durable en ce qui concerne la gestion du sens. Quatre personnes de l'entreprise se sont inscrites à notre programme de formation. Avec le début du coaching à l'automne, nous pourrons bientôt jeter les bases d'une recherche plus systématique afin d'analyser les impacts de cette pratique novatrice sur l'entrée et le suivi des familles dans le difficile travail du deuil. À plus long terme, nous aimerions "exporter», voire transférer notre expertise à d'autres entreprises funéraires.

\section{Bibliographie}

BACQUÉ, Marie Frédérique (1997). Mourir aujourd'hui; les nouveaux rites funéraires, Paris, Odile Jacob.

DES AULNIERS, Luce (1991). "Des rites contemporains en attente de leur vérité », Liturgie, Foi et Culture, vol. 25, n 126, juin, p. 11-19.

HOUDE, Audrey (2004). Le travail de production et de reconstruction des mises en scènes rituelles dans le contexte de la ritua- lité funéraire, mémoire présenté à la Faculté des études supérieures de l'Université de Montréal, programme offert en extension à l'UQAC, octobre 2004.

JOURDENAIS, Manon (1998). Maintenant que je ne vais plus mourir, Montréal, Fides.

ST-ONGE, Sébastien (2001). L'industrie de la mort, Montréal, Nota Bene.

THOMAS, Louis-Vincent (1985). Rites de mort: pour la paix des vivants, Paris, Fayard.

\section{Notes}

1. Il existe un débat important autour de la définition du spirituel. Pour les fins de cet article, nous reprenons les travaux de Manon Jourdenais publiés dans Maintenant que je ne vais plus mourir (Montréal, Fides, 1998) où elle définit la spiritualité autour de six axes qui sont profondément imbriqués: identité et unification de soi, quête de sens, relation avec un absolu, valeurs, appartenance à une communauté, et expressions symboliques et sociales à travers les rites.

2. Sur cet aspect de la séquence tripartite des rites de passage, voir l'article que nous avons déjà publié «Des rites funéraires pour mieux vivre et faire vivre la dernière traversée», Frontières, vol. 16, $\mathrm{n}^{\mathrm{O}} 2$, printemps 2004, p. 53-57.

3. Extrait du dépliant d'information de la Coopérative funéraire de Chicoutimi, cet extrait a été rédigé par monsieur Harold Bouchard, artistre verrier, Atelier à l'An verre. 